\title{
Trastornos autonómicos en enfermedad de Parkinson
}

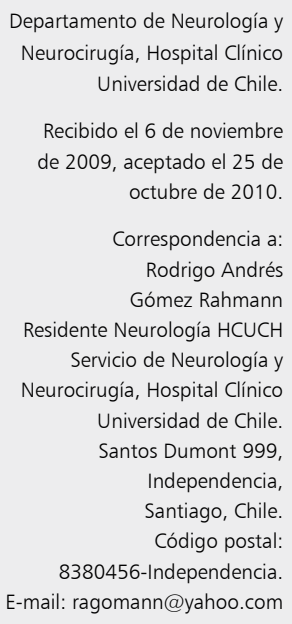

\author{
RODRIGO GÓMEZ, LORENA HUDSON, PABLO VENEGAS
}

\section{Autonomic disturbances in Parkinson disease}

The current concept of Parkinson Disease comprises a group of non-motor symptoms. Among these, dysautonomia is a common problem that deteriorates the quality of life of patients. In this article we review the most common dysautonomic manifestations that are observed in cardiovascular, gastrointestinal, urinary, genital and skin systems. Their possible role as risk factors, premotor symptoms and their implications in the pathogenesis of Parkinson Disease are discussed. A general approach to the main syndromes, based in the available evidence and in our experience is also presented.

(Rev Med Chile 2011; 139: 100-106).

Key words: Autonomic nervous system diseases; Parkinson disease; Primary dysautonomias.
$\mathrm{E}$ n la última década los avances en el conocimiento de la enfermedad de Parkinson (EP) han dejado atrás el paradigma centrado sólo en la afectación motora secundaria a la degeneración de la sustancia nigra, avanzando hacia el concepto de un trastorno caracterizado por la presencia de disautonomía, dissomnia, disfunción motora y demencia.

Las primeras observaciones clínicas de alteraciones del sistema nervioso autónomo (SNA) en EP las hizo el mismo James Parkinson, quien en su monografía original ya describía la disfagia, la sialorrea y la constipación ${ }^{1}$. La correlación anatomopatológica de estas observaciones, sin embargo, debió esperar más de 150 años, hasta la década de los 80’s del siglo pasado, cuando se demostró la presencia de cuerpos de Lewy en el sistema nervioso entérico $(\mathrm{SNE})^{2,3}$. Investigaciones más recientes han demostrado que la progresión patológica de la EP se inicia en dependencias del SNA: nervios y ganglios autonómicos periféricos y núcleo dorsal motor del vago (estadíos 0 y 1 de Braak), siendo el compromiso de la sustancia nigra más tardío (estadío 3 de Braak). En los estadíos tardíos (5 y 6 de Braak) aparece la afectación neocortical ${ }^{4}$. En las Tablas 1 y 2 se presentan, respectivamente, los estadíos de Braak y su correlación con las mani- festaciones no motoras de la EP.

Se ha encontrado cuerpos de Lewy y/o inclusiones de $\alpha$-sinucleína en el sistema nervioso autónomo tanto a nivel central (hipotálamo, asta intermediolateral de médula espinal, núcleo dorsal motor del vago) como periférico (ganglios simpáticos, sistema nervioso entérico, plexos periféricos) siendo, en la EP, más significativo el compromiso de este último ${ }^{2,3-9}$.

La expresión clínica de estos hallazgos es muy amplia y puede afectar a cualquier órgano inervado por el SNA: gastrointestinal, cardiovascular, génito-urinario, piel, glándulas suprarrenales. Estas alteraciones pueden estar presentes al momento del diagnóstico e incluso precederlo (en concordancia con los hallazgos patológicos expuestos) y no se ha encontrado una correlación constante en los estudios entre la aparición de disautonomía y otras variables de la enfermedad (tiempo de evolución, UPDRS motor, trastornos del ánimo), salvo aparentemente la edad del paciente ${ }^{10,11}$.

\section{Epidemiología}

La prevalencia de trastornos autonómicos en EP varía de 14 al 80\% en distintos estudios y según el criterio utilizado. La serie de Verbaan comparó 420 
Trastornos autonómicos en Parkinson - R. Gómez et al

Tabla 1. Estadíos de progresión de EP propuestos por Braak

\begin{tabular}{|cl|}
\hline Estadío & Afectación patológica \\
0 & Nervios y ganglios autonómicos periféricos \\
1 & $\begin{array}{l}\text { Etapa } 0+\text { núcleos olfatorios y bulbo raquí- } \\
\text { deo (núcleo dorsal motor del vago) }\end{array}$ \\
2 & $\begin{array}{l}\text { Etapa } 1+\text { puente (locus ceruleus, núcleo } \\
\text { pedúnculopontino) }\end{array}$ \\
3 & $\begin{array}{l}\text { Etapa } 2+\text { mesencéfalo (substancia nigra } \\
\text { pars compacta, núcleos del rafe) }\end{array}$ \\
4 & $\begin{array}{l}\text { Etapa } 3+\text { prosencéfalo basal (núcleo basal } \\
\text { de Meynert) y mesocortex }\end{array}$ \\
5 & $\begin{array}{l}\text { Etapa } 4+\text { neocorteza de asociación y pre- } \\
\text { frontal }\end{array}$ \\
6 & $\begin{array}{l}\text { Etapa } 5+\text { neocorteza motora y sensitiva } \\
\text { primarias }\end{array}$ \\
\hline
\end{tabular}

Tabla 2. Trastornos no motores de la EP y su correlación con los estadíos de Braak

\begin{tabular}{|lc|}
\hline Manifestación no motora de EP & $\begin{array}{c}\text { Estadío (s) } \\
\text { de Braak }\end{array}$ \\
\hline $\begin{array}{l}\text { Hiposmia (alteraciones en detección, } \\
\text { identificación y discriminación de olores) }\end{array}$ & 1 \\
\hline Alteraciones autonómicas & 0,1 \\
\hline Depresión & 2,3 \\
\hline Trastorno conductual del sueño REM & 2 \\
\hline $\begin{array}{l}\text { Deterioro cognitivo - Demencia } \\
\text { Otros: fatiga, alteración en la visión de } \\
\text { colores, dolor, apatía }\end{array}$ & $4,5,6$ \\
\hline
\end{tabular}

pacientes con EP con 140 controles mediante una encuesta de autorreporte (SCOPA-AUT) y encontró diferencias significativas en todos los dominios del SNA estudiados: gastrointestinal, urinario, cardiovascular, termorregulación, sexual ${ }^{12}$. Magerkurth C et al reportaron presencia de síntomas significativos en la calidad de vida en el 50\% de 141 pacientes encuestados, destacando la disfunción vesical (54\%), el ortostatismo $(53 \%)$ y la constipación $(41 \%)^{13}$.

\section{Sistema cardiovascular}

La hipotensión ortostática (HO) es la manifestación más frecuente, con una incidencia que en general se estima entre 20 y $50 \%$. Más aún,
Goldstein estudió retrospectivamente un grupo de 35 pacientes con $\mathrm{EP}+\mathrm{HO}$ y encontró que $60 \%$ de ellos manifestó esta última precozmente (antes de 1 año de evolución de la EP), en 4 pacientes la $\mathrm{HO}$ precedió a la EP y en 4 dominó la presentación clínica por sobre el parkinsonismo. En estos casos se descartó otras causas de disautonomía, como Atrofia Multisistémica, mediante evaluación clínica y PET cardiaco con MBIG (ver más adelante) ${ }^{10}$. De este modo la $\mathrm{HO}$ se debe sospechar incluso en pacientes jóvenes y con EP de corta evolución.

En los pacientes EP con $\mathrm{HO}$ se ha demostrado una disminución de la inervación simpática cardiaca, una disminución de los niveles plasmáticos de catecolaminas en la posición de pie y una alteración de la respuesta barorrefleja en cuanto a frecuencia cardiaca y vasoconstricción periférica ${ }^{14,15}$. Todo esto lleva a que, en posición de pie, exista una caída progresiva de la presión arterial con un aumento modesto de la frecuencia cardiaca, lo que finalmente resulta en insuficiencia de flujo cerebral.

El diagnóstico requiere un alto nivel de sospecha y una entrevista detallada. Debemos considerar que hasta un tercio de los pacientes pueden no referir síntomas aun con caídas de presión ortostática presincopales $(<80 / 50 \mathrm{mmHg})$ y que en el grupo de pacientes $>70$ años el $30 \%$ no recuerda una caída en los últimos 3 meses, aún habiendo sido presenciada por un familiar ${ }^{16,17}$. Asimismo, debemos dar importancia incluso a síntomas en apariencia vagos (desconcentración, sensación de cabeza abombada, visión borrosa, etc) que se asocian a períodos a veces prolongados de posición de pie (más de 15 minutos en algunos casos) ${ }^{17,20}$.

La hipotensión ortostática se define con una caída sostenida de PA $>20 \mathrm{mmHg}$ sistólica o más de $10 \mathrm{mmHg}$ la diastólica, tras al menos $3 \mathrm{mi}-$ nutos de ponerse de pie $^{18}$. Sin embargo, en EP la caída sistólica puede ser mayor a $100 \mathrm{mmHg}$ y la hipertensión supina no descarta el diagnóstico. Un ejemplo frecuente en el policlínico es un paciente con PA del orden de 170/90 que cae a 70/40 a los 3 minutos de pie, sin que manifieste necesariamente sintomatología asociada.

El examen de elección es el tilt test ${ }^{18}$. A nuestro juicio, sin embargo, la prueba de ortostatismo en la cabecera del paciente mantiene su valor, aunque tomando en cuenta su sensibilidad limitada a la hora de interpretarlo. El examen de detección de emisión de fotón único (PET) con el trazador 
metaiodobenzilguanidina (MIBG) resulta de utilidad para diferenciar una EP con componente $\mathrm{HO}$ precoz de una Atrofia Multisistémica, demostrando una disminución de captación desde etapas tempranas en EP, lo que no ocurre en casos de Atrofia Multisistémica. Por lo mismo, este examen ha sido postulado como biomarcador de detección precoz de $\mathrm{EP}^{19}$.

El tratamiento debe considerar equilibrar presiones supinas con presiones de bipedestación. Resulta de utilidad el autoregistro de presión supina y de pie, así como el tiempo desde posición de pie hasta la aparición de síntomas importantes.

Para el manejo de HO en EP se ha postulado un sinnúmero de medidas generales como aumentar aporte de sal (2 a 8 g/día $\rightarrow$ Na urinario $>140$ $\mathrm{mEq} / 24 \mathrm{~h}$ ), medias compresivas (40-50 $\mathrm{mmHg}$ ), ingesta de agua $>500 \mathrm{ml}$ (aumentaría PA 25-60 $\mathrm{mmHg}$ por 1 hora) y ejercicios específicos, entre otros, ninguno de los cuales ha demostrado uti$\operatorname{lidad}^{20}$. De todos modos parece útil recomendar actividad física moderada y dentro de esto la natación resulta especialmente beneficiosa.

Existen escasos ensayos clínicos controlados que evalúen la efectividad de las terapias farmacológicas específicamente en pacientes EP. Schoffer comparó manejo general, domperidona (30 mg al día) y fludrocortisona ( $0,1 \mathrm{mg}$ al día) en 17 pacientes EP con HO. Se utilizó una escala de reporte subjetivo por parte del paciente y el examinador, y el "tilt test". Los autores describen que tanto domperidona como fludrocortisona disminuyen significativamente las molestias, mientras que en el "tilt test" la domperidona tiene una tendencia a disminuir más efectivamente la caída de presión $\operatorname{arterial}^{20}$. Su mecanismo de acción sería bloqueando el efecto de la dopamina a nivel periférico, la cual a su vez compite con la noradrenalina en el sistema nervioso simpático. En nuestra experiencia la domperidona ha resultado de utilidad con mínimos efectos adversos. Por otro lado, Singer et al evaluaron el efecto de piridostigmina en dosis única matinal de $60 \mathrm{mg}$ en 17 pacientes con $\mathrm{HO}$ neurogénica, 3 de ellos con EP; los resultados del estudio fueron significativos en cuanto a un incremento en la presión ortostática en relación a placebo y una disminución en la caída de presión arterial en el "tilt test", con disminución de los síntomas de ortostatismo y sin incremento en la presión arterial supina ${ }^{21}$. La piridostigmina aumenta la disponibilidad de acetilcolina en la neurona simpática preganglionar, aumentando de esta manera la liberación de catecolamina por parte de la neurona post ganglionar. El efecto neto es un aumento de la resistencia vascular periférica.

El resto de las opciones no han sido sistemáticamente evaluadas en pacientes con $\mathrm{HO}$ asociada a EP. El alfa-agonista midodrina en dosis de 1,25-20 mg, 2 a 3 tomas mantiene su efecto por aproximadamente 4 horas. Se ha demostrado su utilidad en cuadros de $\mathrm{HO}$ neurogénica no $\mathrm{EP}$, tales como falla autonómica primaria ${ }^{22}$. Sin embargo, su uso requiere cierta precaución en pacientes con antecedentes de hipertensión. Los antidepresivos duloxetina y venlafaxina producen una activación indirecta de receptores alfa y aparecen como una opción interesante en pacientes EP con trastornos del ánimo e HO. La fludrocortisona en dosis bajas (0,1 mg al día) produce una sobrexpresión de receptores alfa en la vasculatura y su uso conjunto con midodrina tendría un efecto sinérgico; en dosis de 0,1-0,4 mg/d actúa como expansor de volumen ${ }^{23}$.

\section{Tracto gastrointestinal}

Los avances en la caracterización del sistema nervioso entérico (SNE) han develado una compleja red neuronal con una riqueza de neurotransmisores comparable al SNC, por lo que algunos autores le han denominado "mini-cerebro" 24 . Se estudia activamente su rol en diversas patologías tales como el síndrome de intestino irritable y resulta muy interesante revisar su relación con la EP.

\section{Disfagia}

Evaluaciones de deglución con fluoroscopía demuestran que ésta está alterada en 79-95\% de los pacientes con EP, ocurriendo disfagia en un $50-80 \%$ en algún momento de la evolución. Se ha asociado con alteraciones en la fase preoral y oral (rigidez mandibular, alteraciones motoras linguales, cervicales y de extremidades), con el retraso de la fase faríngea de la deglución y con la hipertonía del esfínter esofágico superior ${ }^{25-28}$. Clínicamente es importante descartar reflujo gastro-esofágico como causa tratable ${ }^{29}$. Respecto al manejo, el efecto de los agentes dopaminérgicos es sólo parcial y se puede considerar el manejo fonoaudiológico $y$, en fases avanzadas, la gastrostomía ${ }^{30,31}$. Como recomendación general es importante una dieta blanda, con espesantes de líquidos, indicando a 
los pacientes con fluctuaciones motoras comer en los períodos "on".

En relación a la sialorrea es importante recalcar que es una consecuencia de la disfagia, mientras que la secreción de saliva está normal e incluso disminuida ${ }^{32,33}$. Por lo mismo, el tratamiento con anticolinérgicos es inefectivo. La toxina botulínica (parótida, submandibular) podría disminuir la salivación pero es técnicamente compleja pudiendo, por otra parte, aumentar la disfagia y la probabilidad de infecciones de la cavidad bucal ${ }^{34}$.

\section{Alteraciones gástricas}

El compromiso se manifiesta principalmente como gastroparesia (36-88\%) con náuseas, saciedad precoz y disconfort abdominal ${ }^{35,36}$. El retraso del vaciamiento se ha implicado en casos de fluctuaciones motoras ("on" retardado y no "on"), siendo de utilidad el manejo con prokinéticos (domperidona) $)^{37,38}$. En casos severos debe considerarse el uso de levodopa gel vía duodenostomía (Duodopa ${ }^{\circledast}$ ), apomorfina subcutánea y/o parches de rotigotina ${ }^{39}$.

La infección por Helicobacter Pylori se ha asociado a disminución de la absorción de L-dopa. El mecanismo mediante el cual este fenómeno se produce no es del todo comprendido. Sin embargo, se ha relacionado con lesiones erosivas gástricas y alcalinización en la zona ${ }^{40}$.

Interesantemente, Braak demostró, en un estudio de necropsias, la presencia de inclusiones inmunorreactivas de $\alpha$-sinucleína en la mucosa gástrica (plexo submucoso de Meissner) de todos los pacientes EP (versus ningún control). Dos de los casos aún no tenían compromiso de sustancia nigra ni síntomas motores. Dichas inclusiones se encontraban a escasos micrones del epitelio gástrico. En base a esta y otras observaciones los autores plantean la hipótesis de un rol etiopatogénico de algún agente químico o infeccioso que, vía mucosa gástrica, alcance el plexo entérico y finalmente de manera retrógrada el SNC ${ }^{41,42}$.

\section{Constipación}

Más de $80 \%$ de los casos con EP tiene un tránsito colónico anormalmente prolongado, existiendo además en mayor o menor grado alteraciones de la relajación anal (disinergia del piso pélvico) ${ }^{25,43,44}$. Clínicamente se presenta constipación en el 30$60 \%$ de los pacientes, frente a lo cual los fármacos dopaminérgicos y anticolinérgicos no son efecti- vos e incluso pueden empeorar el problema. El tratamiento consiste en pesquisar y discontinuar los fármacos que induzcan constipación, manejo dietario y agentes específicos: laxantes osmóticos (psyllium), soluciones de polietilenglicol $17 \mathrm{~g}$ al día, tegaserod 6 mg 2 veces al día. Para enfrentar el problema de la disinergia podría ser de utilidad la apomorfina y el biofeedback ${ }^{45-47}$.

La constipación se ha propuesto como síntoma premotor y como factor de riesgo para desarrollo de EP. Abbott publicó, en el contexto del estudio poblacional Honolulu Heart Program, el seguimiento de 6.790 hombres entre 51 y 75 años sin EP por más de 24 años. Encontró que aquellos hombres con $<1$ deposición al día tenían 2,7 veces más riesgo de desarrollar EP versus aquellos con 1 deposición al día. El riesgo relativo se incrementó 4,1 y 4,7 veces comparado con hombres con 2 deposiciones y $>2$ deposiciones al día, respectivamente $^{48}$. Queda por aclarar si esto representa un síntoma premotor o un factor de riesgo (quizá la mayor permanencia de algún "agente nocivo" en el sistema gastrointestinal).

\section{Tracto genitourinario}

\section{Trastornos urinarios}

Los trastornos urinarios se cuentan entre los más frecuentes $(21-71 \%$ en pacientes EP). La forma clínica predominante es la irritativa (hiperreflexia del detrusor), siendo el síntoma más común la nicturia. También se reporta urgencia, aumento de frecuencia miccional, urgincontinencia ${ }^{49,50}$. Con frecuencia se presentan dificultades en cuanto a la atribución de los síntomas, considerando la alta frecuencia de patología prostática y de insuficiencia del piso pélvico en este grupo etáreo, por lo que los casos que se aparten del patrón habitual deben recibir consulta urológica.

Como opciones de manejo farmacológico tenemos anticolinérgicos como oxibutinina (en Chile Urazol ${ }^{\circledR}$ o Uricort $\left.{ }^{\circledR}\right) 5 \mathrm{mg} 1$ a 3 veces al día, teniendo precaución con sus efectos cognitivos, o antagonistas alfa (terazosina, tamsulozina) [50]. Los agentes dopaminérgicos han mostrado resultados contradictorios, mientras que la cirugía de estimulación profunda núcleo subtalámico ha dado buenos resultados, apoyando la evidencia de que estas alteraciones tendrían un origen predominantemente en el SNC, vía desinhibición del músculo detrusor ${ }^{52,53}$. 


\section{Disfunción sexual}

En hombres se presenta disfunción eréctil hasta en $60 \%$, también retraso o inhibición de la eyaculación. En la mujer puede producirse anorgasmia. Siempre debemos considerar la posibilidad de una depresión concomitante o la presencia de algún fármaco que altere la función eréctil. Descartado esto, la respuesta al sildenafil es satisfactoria. La levodopa puede producir hipersexualidad, especialmente en el contexto de un síndrome de desregulación dopaminérgica.

Xao et $\mathrm{al}^{54}$ estudiaron de forma retrospectiva, en el contexto de un estudio poblacional que observó a 32.616 hombres por 14 años, el riesgo de desarrollar EP en presencia de disfunción eréctil. Encontraron, una vez ajustado por otros factores, que aquellos hombres que al inicio del estudio tenían una disfunción eréctil tuvieron 3,8 veces más probabilidades de desarrollar una EP durante el período de seguimiento.

\section{Alteraciones sudomotoras}

La hiperhidrosis se observa en $30-50 \%$ de los pacientes. Sin embargo, es necesario aclarar que asimismo existen pacientes con sudoración normal y aun disminuida. También se describen alteraciones en la termorregulación. La aparición de dichas alteraciones no se correlaciona con la progresión de la enfermedad. Desde el punto de vista patológico se ha encontrado en biopsias de piel una denervación autónoma de elementos cutáneos, especialmente en glándulas sudoríparas y vasos sanguíneos. Clínicamente los pacientes presentan sudoración excesiva, predominantemente en cara y tronco, lo que puede llegar a ser muy molesto motivando frecuentes mudas de ropa, asociada especialmente a los períodos de "off". La levodopa disminuiría estas molestias, fundamentalmente las asociadas a "off" $55-57$ y se ha planteado también toxina botulínica cuando la molestia es más bien local. La respuesta al tratamiento, en nuestra experiencia, no es satisfactoria.

\section{Conclusión}

Los trastornos autonómicos en EP son un problema precoz, frecuente y que altera significativamente la calidad de vida de los pacientes, por lo que deben ser sospechados y manejados con el mismo ahínco que los síntomas motores. A la fecha resta mucho por precisar en cuanto a los mecanismos implicados, la correlación clínica y el tratamiento adecuado de estas alteraciones. También está por definir su importancia como factores de riesgo, síntomas premotores e incluso su rol en la etiopatogenia de la EP.

\section{Referencias}

1. Parkinson J. An Essay on the Shaking Palsy. London: Whittingham and Rowland 1817.

2. Yoshimura N, Shoji M, Matsui T. An autopsy case of Parkinson's disease manifesting hyperphagia and dysphagia followed by severe achalasia (disorder of motility) of the esophagus. No To Shinkei 1982; 34: 741-6.

3. Qualman SJ, Haupt HM, Yang P, Hamilton SR. Esophageal Lewy bodies associated with ganglion cell loss in achalasia. Similarity to Parkinson's disease. Gastroenterology 1984; 87: 848-56.

4. Braak H, Del Tredici K, Rüb U, de Vos RA, Jansen Steur EN, Braak E. Staging of brain pathology related to sporadic Parkinson's disease. Neurobiol Aging 2003; 24: 197-211.

5. den HJ, Bethlem WJ. The distribution of Lewy bodies in the central and autonomic nervous systems in idiopathic paralysis agitans. J Neurol Neurosurg Psychiatry 1960; 23: 283-90.

6. Eadie MJ. The Pathology of Certain Medullary Nuclei in Parkinsonism. Brain 1963; 86: 781-792.

7. Langston JW, Forno LS. The hypothalamus in Parkinson disease. Ann Neurol 1978; 3: 129-33.

8. Nakajima R, Takahashi K, Nakamura H, Otomo E, Kameyama M. A quantitative study on the intermediolateral cells of the thoracic cord in degenerative diseases of the nervous system (author's transl). Rinsho Shinkeigaku 1981; 21: 581-6.

9. Ohama E, Ikuta F. Parkinson's disease: distribution of Lewy bodies and monoamine neuron system. Acta Neuropathol 1976; 34: 311-319.

10. Goldstein DS. Orthostatic hypotension as an early finding in Parkinson's disease. Clin Auton Res 2006; 16: 46-54.

11. Korchounov A, Kessler KR, Yakhno NN, Damulin IV, Schipper HI. Determinants of autonomic dysfunction in idiopathic Parkinson's disease. J Neurol 2005; 252: 1530-6.

12. Verbaan DJ, Visser M, van Rooden SM, Stiggelbout AM, van Hilten JJ. Patient-reported autonomic symptoms in Parkinson disease. Neurology 2007; 69: 333-41. 
13. Magerkurth CR, Schnitzer, Braune S. Symptoms of autonomic failure in Parkinson's disease: prevalence and impact on daily life. Clin Auton Res 2005; 15: 76-82.

14. Goldstein DS, Eldadah BA, Holmes C, Pechnik S, Moak J, Saleem A et al. Neurocirculatory abnormalities in Parkinson disease with orthostatic hypotension: independence from levodopa treatment. Hypertension 2005; 46: 1333-9.

15. Goldstein DS, Holmes C, Li ST, Bruce S, Metman LV, Cannon RO 3rd. Cardiac sympathetic denervation in Parkinson disease. Ann Intern Med 2000; 133: 338-47.

16. Cummings SR, Nevitt MC, Kidd S. Forgetting falls. The limited accuracy of recall of falls in the elderly. J Am Geriatr Soc 1988; 36: 613-6.

17. Arbogast SD, Alshekhlee A, Hussain Z, McNeeley K, Chelimsky TC. Hypotension unawareness in profound orthostatic hypotension. Am J Med 2009; 122: 574-80.

18. Consensus statement on the definition of orthostatic hypotension, pure autonomic failure, and multiple system atrophy. The Consensus Committee of the American Autonomic Society and the American Academy of Neurology. Neurology 2006; 46: 1470.

19. Takatsu H, Nishida H, Matsuo H, Watanabe S, Nagashima K, Wada H, et al. Cardiac sympathetic denervation from the early stage of Parkinson's disease: clinical and experimental studies with radiolabeled MIBG. J Nucl Med 2000; 41: 71-7.

20. Schoffer KL, Henderson RD, O'Maley K, O’Sullivan JD. Nonpharmacological treatment, fludrocortisone, and domperidone for orthostatic hypotension in Parkinson's disease. Mov Disord 2007; 22: 1543-9.

21. Singer WT, Opfer-Gehrking L, McPhee BR, Hilz MJ, Bharucha AE, Low PA. Acetylcholinesterase inhibition: a novel approach in the treatment of neurogenic orthostatic hypotension. J Neurol Neurosurg Psychiatry 2003; 74: 1294-8.

22. Kaufmann H, Brannan T, Krakoff L, Yahr MD, Mandeli J. Treatment of orthostatic hypotension due to autonomic failure with a peripheral alpha-adrenergic agonist (midodrine). Neurology 1998; 38: 951-6.

23. Davies IB, Bannister RG, Sever PS, Wilcox CS. Fludrocortisone in the treatment of postural hypotension: altered sensitivity to pressor agents [proceedings]. Br J Clin Pharmacol 1978; 6: 444-5.

24. Goyal RK, Hirano I. The enteric nervous system. N Engl J Med 1996; 334: 1106-15.

25. Edwards LL, Quigley EM, Harned RK, Hofman R, Pfeiffer RF. Characterization of swallowing and defecation in Parkinson's disease. Am J Gastroenterol 1994;89(1): 15-25.

26. Leopold NA, Kagel MC. Pharyngo-esophageal dysphagia in Parkinson's disease. Dysphagia 1997; 12: 11-8; discussion 19-20.

27. Leopold NA, Kagel MC. Prepharyngeal dysphagia in Parkinson's disease. Dysphagia 1996; 11: 14-22.

28. Ali GN, Wallace KL, Schwartz R, DeCarle DJ, Zagami AS, Cook IJ. Mechanisms of oral-pharyngeal dysphagia in patients with Parkinson's disease. Gastroenterology 1996; 110: 383-92.

29. Byrne KG, Pfeiffer R, Quiqley EM. Gastrointestinal dysfunction in Parkinson's disease. A report of clinical experience at a single center. J Clin Gastroenterol 1994; 19: 11-6.

30. Monte FS, da Silva-Junior FP, Braga-Neto P, Nobre MA, de Bruin VM. Swallowing abnormalities and dyskinesia in Parkinson's disease. Mov Disord 2005; 20: 457-62.

31. Deane KH, Whurr R, Clarke CE, Playford ED, Ben-Shlomo Y. Non-pharmacological therapies for dysphagia in Parkinson's disease. Cochrane Database Syst Rev 2001; (1): CD002816.

32. Bagheri H, Damase-Michel C, Lapeyre-Mestre M, Cismondo S, O'Connell D, Senard JM, et al. A study of salivary secretion in Parkinson's disease. Clin Neuropharmacol 1999; 22: 213-5.

33. Proulx M, de Courval FP, Wiseman MA, Panisset M. Salivary production in Parkinson's disease. Mov Disord 2005; 20: 204-7.

34. Ondo WG, Hunter C, Moore W. A double-blind placebocontrolled trial of botulinum toxin B for sialorrhea in Parkinson's disease. Neurology 2004; 62: 37-40.

35. Hardoff R, Sula M, Tamir A, Soil A, Front A, Badarna $S$, et al. Gastric emptying time and gastric motility in patients with Parkinson's disease. Mov Disord 2001; 16: 1041-7.

36. Goetze O, Nikodem AB, Wiezcorek J, Banasch M, Przuntek $\mathrm{H}$, Mueller T, et al. Predictors of gastric emptying in Parkinson's disease. Neurogastroenterol Motil 2006; 18: 369-75.

37. Djaldetti R, Baron J, Melamed E. Gastric emptying in Parkinson's disease: patients with and without response fluctuations. Neurology 1996; 46: 1051-4.

38. Kurlan R, Rothfield KP, Woodward WR, Nutt JG, Miller C, Lichter D, et al. Erratic gastric emptying of levodopa may cause "random" fluctuations of parkinsonian mobility. Neurology 1988; 38: 419-21.

39. Kurlan R, Nutt JG, Woodward WR, Rothfield K, Lichter D, Miller C, et al. Duodenal and gastric delivery of levodopa in parkinsonism. Ann Neurol 1988; 23: 589-95.

40. Pierantozzi M, Pietroiusti A, Brusa L, Galati S, Stefani A, Lunardi G, et al. Helicobacter pylori eradication and 1-dopa absorption in patients with PD and motor fluctuations. Neurology 2006; 66: 1824-9. 
41. Braak H, de Vos RA, Bohl J, Del Tredici K. Gastric alphasynuclein immunoreactive inclusions in Meissner's and Auerbach's plexuses in cases staged for Parkinson's disease-related brain pathology. Neurosci Lett 2006; 396: 67-72.

42. Braak H, Rüb U, Gai WP, Del Tredici K. Idiopathic Parkinson's disease: possible routes by which vulnerable neuronal types may be subject to neuroinvasion by an unknown pathogen. J Neural Transm 2003; 110: 517 36.

43. Edwards LL, Quigley EM, Pfeiffer RF. Gastrointestinal dysfunction in Parkinson's disease: frequency and pathophysiology. Neurology 1992; 42: 726-32.

44. Mathers SE, Kempster PA, Law PJ, Kramkel JP, Bartram CI, Lees AJ, et al. Anal sphincter dysfunction in Parkinson's disease. Arch Neurol 1989; 46: 1061-4.

45. Jost WH. Gastrointestinal motility problems in patients with Parkinson's disease. Effects of antiparkinsonian treatment and guidelines for management. Drugs Aging 1997; 10: 249-58.

46. Astarloa R, Mena MA, Sánchez V, de la Vega L, de Yébenes JG. Clinical and pharmacokinetic effects of a diet rich in insoluble fiber on Parkinson disease. Clin Neuropharmacol 1992; 15: 375-80.

47. Ashraf W, Pfeiffer RF, Park F, Lof J, Quigley EM. Constipation in Parkinson's disease: objective assessment and response to psyllium. Mov Disord 1997; 12: 946-51.

48. Abbott RD, Petrovitch H, White LR, Masaki KH, Tanner $\mathrm{CM}$, Curb JD, et al. Frequency of bowel movements and the future risk of Parkinson's disease. Neurology 2001; 57: 456-62.

49. Winge K, Fowler CJ. Bladder dysfunction in Parkinsonism: mechanisms, prevalence, symptoms, and management. Mov Disord 2006; 21: 737-45.

50. Campos-Sousa RN, Quagliato E, da Silva BB, de Carvalho RM Jr, Ribeiro SC, de Carvalho DF. Urinary symptoms in Parkinson's disease: prevalence and associated factors. Arq Neuropsiquiatr 2003; 61 (2B): 359-63.

51. Singer C. Urinary dysfunction in Parkinson's disease. Clin Neurosci 1998; 5: 78-86.

52. Aranda B, Cramer P. Effects of apomorphine and L-dopa on the parkinsonian bladder. Neurourol Urodyn 1993; 12: 203-9.

53. Herzog J, Weiss PH, Assmus A, Wefer B, Seif C, Braun $\mathrm{PM}$, et al. Improved sensory gating of urinary bladder afferents in Parkinson's disease following subthalamic stimulation. Brain 2008; 131(Pt 1): 132-45.

54. Gao X, Chen H, Schwarzschild MA, Glasser DB, Logroscino G, Rimm EB. Erectile function and risk of Parkinson's disease. Am J Epidemiol 2007; 166: 1446-50.

55. Hirayama M. Sweating dysfunctions in Parkinson's disease. J Neurol 2006; 253 Suppl 7: VII42-47.

56. Sage JI, Mark MH. Drenching sweats as an off phenomenon in Parkinson's disease: treatment and relation to plasma levodopa profile. Ann Neurol 1995; 37: 120-2.

57. Pursiainen V, Haapaniemi TH, Korpelainen JT, Sotaniemi KA, Myllylä VV. Sweating in Parkinsonian patients with wearing-off. Mov Disord 2007; 22: 828-32. 